\title{
The pathogenesis of endomyocardial fibrosis: the question of 5-hydroxytryptamine
}

\author{
G. O. Ojo \\ From the Department of Physiology, University of Ibadan, Ibadan, Nigeria
}

Thirty Nigerians with established endomyocardial fibrosis were studied. Basal serum 5-hydroxytryptamine levels were estimated on three different occasions at one-monthly intervals. Twentyfour hours before each estimation, dietary staples containing 5-hydroxytryptamine were excluded from the diet. Subsequently the patients were fed on a plantain diet and serum levels of 5-hydroxytryptamine were estimated at one- and three-hour intervals after plantain ingestion. 5-hydroxytryptamine was assayed on the fundal strip of rat's stomach. These values were compared with those obtained in an earlier study on healthy Nigerians. It was observed that, as in healthy Nigerians, no significant increase in serum 5-hydroxytryptamine levels occurred in these patients after plantain ingestion. The difference between endomyocardial fibrosis and carcinoid heart disease is underlined and it is emphasized that no correlation exists between the incidence of endomyocardial fibrosis and the high content of 5-hydroxytryptamine in the local dietary staples.

Endomyocardial fibrosis is one of the commonest heart diseases in many African countries south of the Sahara. Many theories have been put forward regarding its pathogenesis and possible aetiological factors. One such theory is the association between 5-hydroxytryptamine and endomyocardial fibrosis. This association emanated from the similarity between endomyocardial fibrosis and carcinoid heart disease (Ball, 1957; Arnott, 1959) and the presence in large quantities of 5-hydroxytryptamine in plantain and bananas, the major dietary item in some African countries where endomyocardial fibrosis is endemic (West, 1958; Marshall, 1959; Foy and Parratt, 1960; Crawford, 1963). It is presumed that plantain ingestion raises serum 5-hydroxytryptamine levels with consequent damage to the heart. Cardiac damage simulating endomyocardial fibrosis has been reported in guinea-pigs fed on a diet of plantain (McKinney and Crawford, I965). In addition, raised excretion of 5-hydroxyindole-acetic acid, the urinary metabolite of 5-hydroxytryptamine said to be pathognomonic of carcinoid syndrome (Sjoerdsma, Weissbach, and Udenfriend, 1956), is also typical of these Africans after a meal of plantain or bananas (Crawford, 1963; Foy and Parratt, 1962; Ojo and Parratt, I966).

The metabolism of 5-hydroxytryptamine

Received Io February 1970. is however quite rapid in healthy subjects, and raised serum 5-hydroxytryptamine values are not found in healthy Africans after plantain ingestion (Ojo, 1969). If an impairment of 5-hydroxytryptamine metabolism is the factor responsible for the susceptibility of some Africans to endomyocardial fibrosis, then it may be expected that endogenous 5-hydroxytryptamine, having its metabolic pathway blocked, accumulates in the serum, and that serum 5-hydroxytryptamine levels are further increased after plantain ingestion. The present investigation is designed to assess the validity of this hypothesis.

\section{Materials and methods}

Thirty Nigerian patients with established endomyocardial fibrosis were studied. All the patients were free from any severe degree of cardiac decompensation and were brought into hospital solely for the purposes of this investigation. Twenty-four hours before each estimation, articles of food such as tomatoes, pineapples, plantain, and bananas known to contain 5-hydroxytryptamine were excluded from the diet. Determinations of serum 5-hydroxytryptamine were made at one-monthly intervals for three months on every patient, and the mean of these three values constituted the basal serum 5hydroxytryptamine level. Each patient was subsequently fed on a known weight of fried plantain (dodo), and serum 5-hydroxytryptamine levels were determined at one- and three-hour intervals after plantain ingestion. 
Blood samples (Io ml.) were obtained by venepuncture with silicone syringes from each subject. 5-hydroxytryptamine was extracted from each sample by the method of Hardisty and Stacey (1955) and was assayed on the fundal strip of rat's stomach, as described by Vane (1957) with the modification of Lin and Yeoh (1965).

\section{Results}

As shown in the Table, basal serum 5hydroxytryptamine levels in these patients ranged from 0.02 to $0.46 \mu \mathrm{g} . / \mathrm{ml}$. blood. The values for each subject were fairly constant over the three-month period of investigation. The same degree of constancy was obtained in healthy Nigerians (Ojo, 1969). Mean 5-hydroxytryptamine value and standard deviation for all 30 patients was $0.16 \pm 0.1$ $\mu \mathrm{g} . / \mathrm{ml}$. After plantain ingestion, serum 5-hydroxytryptamine levels showed no significant increase, the mean value plus standard deviation being $0.17 \pm 0.11 \mu \mathrm{g} . / \mathrm{ml}$. Calculated weights of ingested 5-hydroxytryptamine contained in the plantain meals ranged from 8.75 to $18.62 \mathrm{mg}$.; the mean was $13.45 \pm \mathrm{I} \cdot 5$ mg. The Fig. illustrates the effect of plantain ingestion on serum 5-hydroxytryptamine levels in these patients.

TABLE Effect of plantain ingestion on serum 5-hydroxytryptamine (5-HT) in 30 patients with endomyocardial fibrosis

\begin{tabular}{lll}
$\begin{array}{l}\text { Basal serum } \\
5-H T(\mu g . / m l .)\end{array}$ & $\begin{array}{l}\text { Calculated weight } \\
\text { of } 5-H T(\mu g . \\
\text { present in } \\
\text { plantain meals) }\end{array}$ & $\begin{array}{l}\text { Serum } 5-H T \\
\text { after plantain } \\
\text { meals }(\mu g . / m l .)\end{array}$ \\
\hline $0.16 \pm 0.1$ & $\begin{array}{l}13.45 \pm 1.5 \\
(8.75-18.62)\end{array}$ & $\begin{array}{l}0.17 \pm 0.11 \\
(0.05-0.48)\end{array}$
\end{tabular}

Values are means \pm standard deviations with range in parentheses.

\section{Discussion}

Mean basal 5-hydroxytryptamine level in 30 healthy Nigerians was $0.22 \pm 0.13 \mu \mathrm{g} . / \mathrm{ml}$. (Ojo, 1969); the corresponding value of $0 \cdot 16 \pm 0 \cdot 1$ for patients with endomyocardial fibrosis is significantly lower at a probability of more than 0.0I. This accords with the diminished basal urinary excretion of 5hydroxyindole-acetic acid in these patients (Ojo and Parratt, 1966). Endogenous production of 5-hydroxytryptamine must therefore be lower in patients with endomyocardial fibrosis than in healthy Nigerians. Endogenous 5-hydroxytryptamine is derived from dietary tryptophane after the processes of hydroxylation and decarboxylation (Sjoerdsma et al., 1956). Pyridoxine phosphate is a coenzyme of

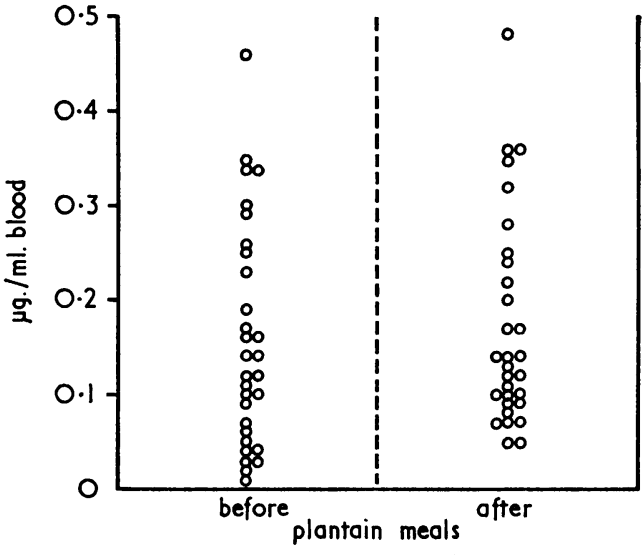

Serum 5-hydroxytryptamine values in patients with endomyocardial fibrosis

5-hydroxytryptophan decarboxylase, the enzyme responsible for the final elaboration of 5-hydroxytryptamine (Buxton and Sinclair, 1956; Bülbring and Lin, 1958). It is particularly significant that endomyocardial fibrosis is more commonly seen among the poor Nigerians in whom protein malnutrition and vitamin deficiency, especially of the $B$ complex group, are common (Abrahams, 1959, 1962). A deficiency of dietary tryptophane and pyridoxine phosphate thus appears to be responsible for the lower endogenous production of 5-hydroxytryptamine in patients with endomyocardial fibrosis. The observation that raised urinary 5-hydroxyindole-acetic acid excretions occur in these patients after plantain meals (Ojo and Parratt, 1966), without a corresponding increase in serum hydroxytryptamine levels, however, indicates that 5-hydroxytryptamine absorbed from plantain rich diets are rapidly converted to urinary 5-hydroxyindole-acetic acid. Therefore deamination of 5-hydroxytryptamine to 5-hydroxyindole-acetic acid is not abnormal in patients with endomyocardial fibrosis. Indeed, the total percentage recovery of exogenous 5hydroxytryptamine as urinary 5-hydroxyindole-acetic acid was similar in both healthy Nigerians and Nigerians with endomyocardial fibrosis (Ojo and Parratt, 1966).

The hypothesis incriminating 5-hydroxytryptamine in the pathogenesis of endomyocardial fibrosis rests on three main points; the assumption that plantain ingestion results in high serum 5-hydroxytryptamine levels in susceptible Africans, the close similarity between endomyocardial fibrosis and carcinoid heart lesion, and finally the observed correlation between 5-hydroxytryptamine in the diet and the prevalence of endomyocardial fibrosis. The first premise is no longer tenable and 
attention will now be focused on the second and third.

A closer scrutiny of endomyocardial fibrosis and carcinoid heart disease reveals that they are different clinico-pathological entities. Damage to the inner third of the myocardium peculiar to endomyocardial fibrosis is absent in carcinoid heart disease. In the former the severest lesions are in the mural endocardium, especially of the apices of the ventricles. Valvular defects are secondary and result from spread of fibrotic process from the mural endocardium. On the contrary, in carcinoid heart disease, the valves bear the major brunt of the damage and constitute the primary lesion. Mural carcinoid plaques occur only in the paths of regurgitant and turbulent flow consequent on the valvular defects. In addition, while it is the delicate veiling of the endocardial inflow tract that is the target in endomyocardial fibrosis, it is the coarser outflow tract where predominant carcinoid plaques are seen. In the carcinoid heart, an atypical fibrous tissue resembling cartilage is superimposed on an intact and relatively healthy endocardium. In contrast to this is the destructive nature of lesions in endomyocardial fibrosis where the normal endocardium is swept away and replaced by a dense fibrous scar (Williams, Ball, and Davies, I954; Fischer and Lindeneg, 1958; Roberts and Sjoerdsma, 1964).

Lesions in the systemic veins and major coronary arteries frequently seen in carcinoid heart disease are rare in endomyocardial fibrosis, while ante-mortem thrombi and embolic complications of endomyocardial fibrosis have not been described in carcinoid heart disease. Indeed, the diverse biological effects of 5-hydroxytryptamine evident in the carcinoid syndrome are not features of endomyocardial fibrosis. Constipation rather than diarrhoea is the aftermath of a heavy plantain meal in Nigerians. Flushing and other vasomotor phenomena do not occur in endomyocardial fibrosis; oliguria and bronchospasm if present are terminal events associated with gross cardiac decompensation. It thus appears extremely unlikely that the different pathological lesions of endomyocardial fibrosis and carcinoid heart are mediated by the same aetiological agent. 5-hydroxytryptamine is definitely not a common denominator in both conditions.

Detailed questionaries presented to Nigerian patients with endomyocardial fibrosis did not confirm that they habitually consumed plantain and bananas. Personal experience reveals that the practice of plantain ingestion is in fact commoner among the sophisticated
Nigerians, for, in the urban areas at least, this item of the diet is relatively expensive. But endomyocardial fibrosis is mainly prevalent in the lower socio-economic stratum of Nigerian society, where diet is confined essentially to the bulkier, cheaper, and more easily accessible cassava diet. Reports from Uganda also indicate that endomyocardial fibrosis is commoner among the immigrant labourers from Rwanda-Burundi who consume much less plantain than the wealthier Baganda people (Shaper and Coles, 1965). Williams (1967) quite rightly asserted that it is untrue that endomyocardial fibrosis and plantain diet share a common geographical distribution or that the incidence of this disease correlates with the plantain eating habits of the African population. Thus endomyocardial fibrosis is rare in the Caribbean where plantain is also consumed in large quantities. Indeed, Antia, Talbert, and Paplanus (1968) denied the existence of cardiac damage in dogs fed on a diet of plantain for nine months.

\section{Conclusion}

The hypothesis that 5-hydroxytryptamine is instrumental in the pathogenesis of endomyocardial fibrosis rests on highly tenuous grounds (van der Geld et al., 1966). This association evolved from the spurious similarity between endomyocardial fibrosis and carcinoid heart disease, the widely accepted correlation between 5-hydroxytryptamine in the diet, and the incidence of endomyocardial fibrosis, as well as the false assumption that plantain ingestion results in chronically high serum 5-hydroxytryptamine levels in the susceptible Africans. Endomyocardial fibrosis and carcinoid heart disease are however different clinico-pathological entities, and high serum levels of 5-hydroxytryptamine do not occur in patients with endomyocardial fibrosis or in normal healthy subjects after a meal of plantain. The theory that 5-hydroxytryptamine may be an aetiological factor in the pathogenesis of endomyocardial fibrosis emanated from scientific conjecture and more speculation, and should now be discarded in the face of overwhelming experimental and clinical data.

I would like to express my gratitude to Dr. A. C. Ikeme, formerly Consultant Cardiologist to the University College Hospital, Ibadan, for permission to study his patients, to Professor John Grayson and Dr. J. R. Parratt, formerly Head of Department and Senior Lecturer, respectively, in the Department of Physiology for their guidance and encouragement, and finally to the Senate Research Grant Committee, University of Ibadan, who provided funds for this project. 


\section{References}

Abrahams, D. G. (1959). An unusual form of heartdisease in West Africa; its relation to endomyocardial fibrosis. Lancet, 2, II I.

- (1962). Endomyocardial fibrosis of the right ventricle. Quarterly fournal of Medicine, 3I, I.

Antia, A. U., Talbert, J. L., and Paplanus, S.H. (1968). Etiology of endomyocardial fibrosis: an experimental evaluation of plantain ingestion and chronic lymphatic obstruction. Fohns Hopkins Medical fournal, 122, 87.

Arnott, W. M. (1959). A problem of tropical cardiology. British Medical fournal, 2, 1273.

Ball, J. D. (1957). Endomyocardial fibrosis. Proceedings of the Royal Society of Medicine, 50, 43.

Bülbring, E., and Lin, R. C. Y. (1958). The effect of intraluminal application of 5-hydroxytryptamine and hydroxytryptophan on peristalsis. The local production of $5-\mathrm{HT}$ and its release in relation to intraluminal pressure and propulsive activity. fournal of Physiology, 140, 38r.

Buxton, J., and Sinclair, H. M. (1956). Pyridoxal phosphate as a coenzyme of 5-hydroxytryptophan decarboxylase. Biochemical fournal, 62, $27 \mathrm{P}$.

Crawford, M. A. (1963). Endomyocardial fibrosis and carcinoidosis. A common denominator ? American Heart fournal, 66, 273.

Fischer, S., and Lindeneg, O. (1958). Cardiac changes in argentaffinomatosis. Acta Pathologica et Microbiologica Scandinavica, 44, 128.

Foy, J. M., and Parratt, J. R. (I960). A note on the presence of noradrenaline and 5-hydroxytryptamine in plantain (Musa sapientum, var paradisiaca). fournal of Pharmacy and Pharmacology, 12, 360.

- , and - (1962). Urinary excretion of 5-hydroxyindoleacetic acid in West Africans. Lancet, $\mathbf{I}$, 942.

Hardisty, R. M., and Stacey, R. S. (1955). 5-Hydroxytryptamine in normal human platelets. Fournal of Physiology, 130, 7I I.

Lin, R. C. Y., and Yeoh, T. S. (1965). An improvement on Vane's stomach strip preparation for the assay of 5-hydroxytryptamine. Fournal of Pharmacy and Pharmacology, 17, 524

McKinney, B., and Crawford, M. A. (1965). Fibrosis in guineapig heart produced by plantain diet. Lancet, 2, 880.

Marshall, P. B. (1959). Catechols and tryptamines in 'matoke' banana (Musa paradisiaca). fournal of Pharmacy and Pharmacology, Ir, 639.

Ojo, G. O. (1969). Plantain meals and serum 5hydroxytryptamine in healthy Nigerians. West African Medical fournal, 18, 174.

-, and Parratt, J. R. (1966). Urinary excretion of 5-hydroxyindoleacetic acid in Nigerians with endomyocardial fibrosis. Lancet, $1,854$.

Roberts, W. C., and Sjoerdsma, A. (1964). The cardiac disease associated with the carcinoid syndrome (carcinoid heart disease). American fournal of Medicine, 36, 5.

Shaper, A. G., and Coles, R. M. (1965). The tribal distribution of endomyocardial fibrosis in Uganda. British Heart fournal, 27, I21.

Sjoerdsma, A., Weissbach, H., and Udenfriend, S. (1956). A clinical, physiologic and biochemical study of patients with malignant carcinoid (Argentaffinoma). American fournal of Medicine, 20, 520.

van der Geld, H., Peetoom, F., Somers, K., and Kanyerezi, B. R. (1966). Immunohistological and serological studies in endomyocardial fibrosis. Lancet, 2, 1210.

Vane, J. R. (1957). A sensitive method for the assay of 5-hydroxytryptamine. British fournal of Pharmacology and Chemotherapy, 12, 344.

West, G. B. (1958). Tryptamine in edible fruits. Fournal of Pharmacy and Pharmacology, 10, 589.

Williams, A. W. (1967). Discussion on Shaper's article 'On the nature of some tropical cardiopathies'. Transactions of the Royal Society of Tropical Medicine and Hygiene, 6r, 477.

_, Ball, J. D., and Davies, J. N. P. (1954). Endomyocardial fibrosis in Africa: its diagnosis, distribution and nature. Transactions of the Royal Society of Tropical Medicine and Hygiene, 48, 290. 